\title{
University of Western Ontario
}

\section{The burden of multimorbidity}

The focus of primary health care (PHC) in developed countries is now largely centred on the treatment and management of long-term or chronic diseases. Due to shared risk factors and interaction among diseases, chronic conditions are increasingly occurring in clusters. ${ }^{1}$ In Canada, more than $50 \%$ of adults aged 65 years and older report having at least two chronic diseases. ${ }^{2}$ The co-occurrence of multiple chronic diseases in an individual, or multimorbidity, is also understood to be the norm rather than the exception in PHC. ${ }^{3}$ Multimorbidity is associated with reduced quality of life, limited functional status, polypharmacy, increased mortality, and high health care costs. ${ }^{3}$ Deemed an "endless struggle" by PHC providers, multimorbidity is becoming more prevalent in younger patients and is no longer confined to elderly populations. ${ }^{1,4}$ This phenomenon is pushing PHC providers and researchers alike to understand its multifaceted nature. A better understanding of the etiology behind multimorbidity can lead to a transformed clinical approach that will, in turn, be cost-saving in the long-run. To achieve this, three main components are necessary.

\section{(1) Defining and measuring multimorbidity}

Due to the relatively recent emergence of research in this field, the definition and measurement of multimorbidity remain inconsistent. While the definition has continually revolved around the co-existence of $\geq 2$ chronic diseases or $\geq 3$ chronic diseases in an individual, no consensus exists to date. Literature shows that $51 \%$ of published multimorbidity research did not explicitly state the definition of multimorbidity used, adding to the mystery. ${ }^{5}$ There is also marked variation in the methodology of multimorbidity studies with respect to the type of data used, the population studied, and the types of chronic diseases included in the pool of multimorbidity. ${ }^{6,7} \mathrm{~A}$ recent comparison of three studies found that the prevalence levels of multimorbidity in a PHC setting ranged from $34 \%$ to $95 \% .{ }^{6}$ Persistent heterogeneity hinders comparability across studies and limits the ability to make informed health system and health policy decisions. ${ }^{6,7}$ Consequently, an explicit and unified methodology to examine multimorbidity in PHC research is needed.

\section{(2) Building our understanding of multimorbidity}

Historically, medical research has focused on patients suffering from single and discrete diseases, such as diabetes or hypertension. However, this single-disease orientation is inapplicable for patients living with multimorbidity because of the interrelatedness of chronic diseases and common underlying causal mechanisms. ${ }^{1,8-9}$ For example, ongoing PHC research both in Canada and abroad is uncovering the non-random clusters of chronic diseases and clinically consistent multimorbidity patterns. ${ }^{1}$ We are also learning of the deeply entangled sociocultural causes of chronic diseases, with the onset of multimorbidity occurring 10 to 15 years earlier in individuals living in deprived areas. ${ }^{10}$ This research is creating a more nuanced understanding of multimorbidity, which will lead to more effective chronic disease prevention and management strategies.

\section{(3) Transforming PHC clinical multimorbidity}

Key elements of our health care system have been organized and designed for patients with discrete diseases, including clinical practice guidelines and disease management strategies for providers. There are well-established calls for these elements to be renewed and restructured. Clinical practice guidelines have the potential to improve care and outcomes for those with chronic disease, but seldom explicitly account for patients with multimorbidity. ${ }^{9}$ Updated clinical guidelines should be reformatted to synthesize the evidence for clinical recommendations, - 
while identifying synergies and contradictions of clinical approaches in the face of multimorbidity. ${ }^{9}$ Although it will admittedly not be possible to provide rigorous evidence for every possible combination of disease, this transformation will require an integrated team of professionals and a change from our traditional single-disease focus, facilitated by a patient-centred approach. Indeed, these demands indicate a significant undertaking and a change in the status quo. However, these new approaches will become much needed resources for our frontline PHC providers and their patients.

\section{Reorienting research and delivery in PHC}

Multimorbidity has set the stage for us to reorient the delivery of PHC. We must harness the growing momentum to conduct more robust PHC research, establish a multidisciplinary and patient-centred approach, and transform the clinical management of multimorbidity in PHC. In the context of PHC research, an explicit and comprehensive definition of multimorbidity will help guide health system design and health policy decision-making. In the context of PHC delivery, there is a need to control or even cope with - the tsunami of demands from these patients. ${ }^{4,6}$ We must reorient our thinking, from a focus on disease to recognizing the needs and goals of the growing number of patients living with multimorbidity. These elements are steps towards adequately responding to this new reality. Not only will this reorientation help our current PHC system cope with the complexities of multimorbidity, it will also help ensure that our system is responsive and sustainable into the future.
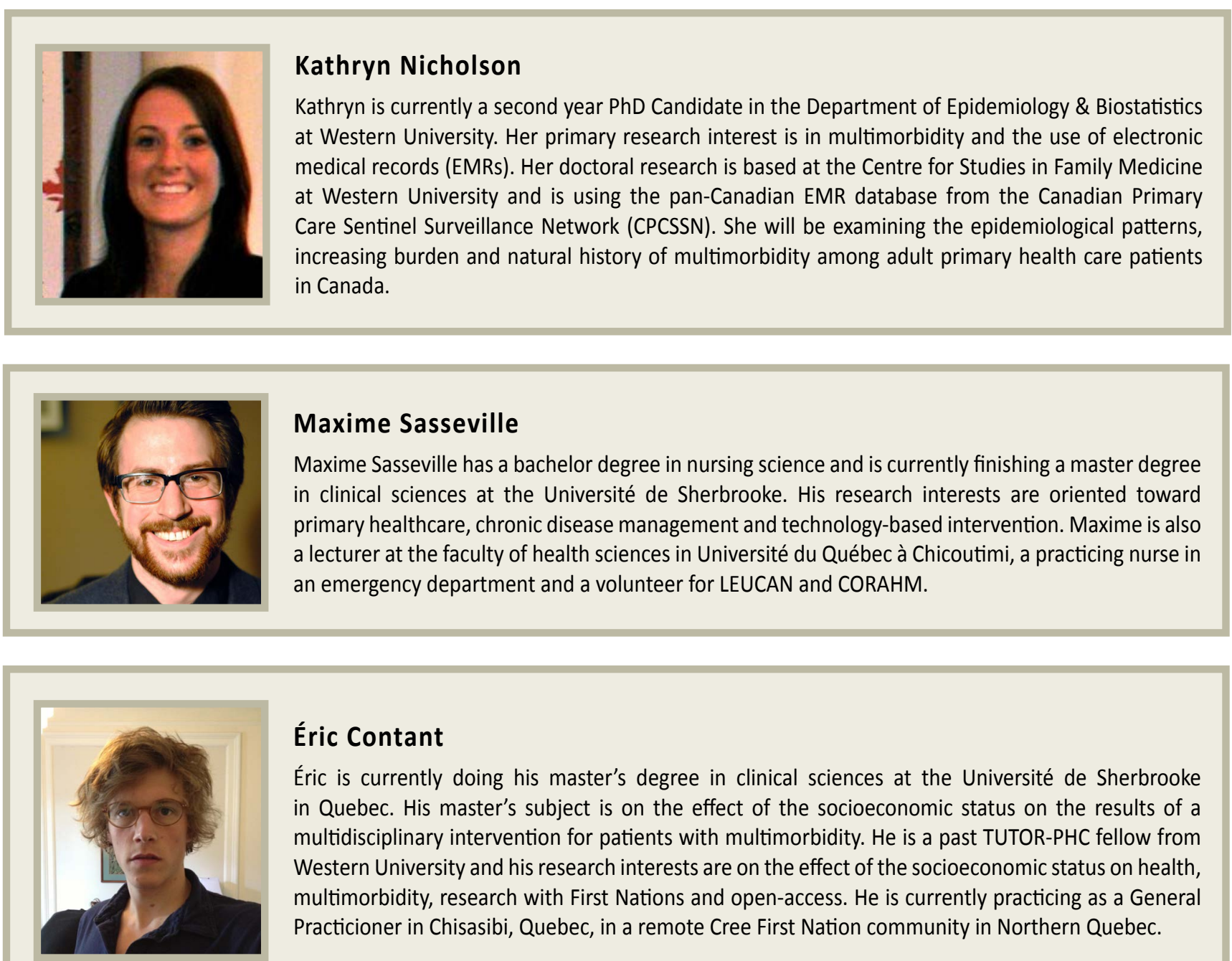

\section{Éric Contant}

Éric is currently doing his master's degree in clinical sciences at the Université de Sherbrooke in Quebec. His master's subject is on the effect of the socioeconomic status on the results of a multidisciplinary intervention for patients with multimorbidity. He is a past TUTOR-PHC fellow from Western University and his research interests are on the effect of the socioeconomic status on health, multimorbidity, research with First Nations and open-access. He is currently practicing as a General Practicioner in Chisasibi, Quebec, in a remote Cree First Nation community in Northern Quebec. 


\section{References}

1. Prados-Torres A, Calderón-Larrañaga A, Hancco-Saavedra J, Poblador-Plou $B$, van den Akker M. Multimorbidity patterns: a systematic review. J Clin Epidemiol. 2014 Mar; 67(3): 254-266.

2. Broemeling AM, Watson DE, Prebtani F. Population patterns of chronic health conditions, co-morbidity and healthcare use in Canada: implications for policy and practice. Healthcare Q. 2008; 11(3): 70-76.

3. Fortin M, Soubhi H, Hudon C, Bayliss EA, van den Akker M. Multimorbidity's many challenges. BMJ. 2007 May; 334(7602): 1016-1017.

4. O'Brien R, Wyke S, Guthrie B, Watt G, Mercer S. An 'endless struggle': a qualitative study of general practitioners' and practice nurses' experiences of managing multimorbidity in socio-economically deprived areas of Scotland. Chronic Illn. 2011 Mar; 7(1): 45-59.

5. Almirall J, Fortin $\mathrm{M}$. The coexistence of terms to describe the presence of multiple concurrent diseases. J Comorbidity. 2013; 3: 4-9.

6. Stewart M, Fortin M, Britt HC, Harrison CM, Maddocks HL. Comparisons of multi-morbidity in family practice - issues and biases. Fam Pract. 2013 Aug; 30(4): 473-480.

7. Fortin $\mathrm{M}$, Stewart $\mathrm{M}$, Poitras $\mathrm{ME}$, Almirall J, Maddocks H. A systematic review of prevalence studies on multimorbidity: toward a more uniform methodology. Ann Fam Med. 2012 Apr; 10(2): 142-151.

8. Starfield B, Kinder K. Multimorbidity and its measurement. Health Policy. 2011 Nov; 103(1): 3-8.

9. Guthrie B, Payne K, Alderson P, McMurdo ME, Mercer SW. Adapting clinical guidelines to take account of multimorbidity. BMJ. 2012 Oct; 345: 6341-6343.

10. Barnett K, Mercer SW, Norbury M, Watt G, Wyke S, Guthrie B. Epidemiology of multimorbidity and implications for health care, research, and medical education: a cross-sectional study. Lancet. 2012 Jul; 380(9836): 37-43. 